\title{
Right atrial fat emboli detection and changes in D-dimer values in reverse shoulder arthroplasty: a comparison with total hip arthroplasty
}

Shusuke Ueda, Toru Ichiseki, Daisuke Soma, Norio Kawahara

Department of Orthopaedic Surgery, Kanazawa Medical University, Ishikawa, Japan

Submitted: 4 December 2018

Accepted: 7 April 2019

Arch Med Sci Civil Dis 2019; 4: e84-e88

DOI: https://doi.org/10.5114/amscd.2019.87003

Copyright @ 2019 Termedia \& Banach

\section{Abstract}

Introduction: The underlying pathophysiology of venous thromboembolism (VTE) in upper limb surgery has been scarcely reported. This prompted us to investigate, using transoesophageal echocardiography (TEE) intraoperatively, the impact on haemodynamics in reverse shoulder arthroplasty (RSA) as compared with total hip arthroplasty (THA).

Material and methods: The subjects comprised five shoulders undergoing RSA (RSA group) in our department. In all cases TEE was performed intraoperatively, with the haemodynamic state of the right atrium and superior vena cava monitored throughout the intraoperative period. As investigated items, blood D-dimer values were measured and compared intraoperatively, on postoperative day 1 , day 4 , week 1 , and week 2 . As a control group, five cases undergoing THA under the same conditions were compared with the RSA group.

Results: Intraoperatively, in both RSA and THA groups, echogenic emboli were identified in the right atrium on TEE during bone reaming and artificial joint insertion. No significant differences were found between the two groups with respect to intraoperative or postoperative D-dimer changes. Also, in neither group was deep vein thrombosis (DVT) or pulmonary embolism (PE) detected either by lower limb venous ultrasonography or lung ventilation-perfusion scintigraphy.

Conclusions: Fat emboli occur as frequently in upper limb surgery as in lower limb surgery, and the possibility of fat embolism syndrome as a postoperative complication needs to be kept in mind. Measurement of D-dimer values is useful in the early detection of DVT in upper limb surgery.

Key words: fat embolism syndrome, fat emboli, transoesophageal echocardiography, reverse shoulder arthroplasty, deep vein thrombosis.

\section{Introduction}

Deep vein thrombosis (DVT) and pulmonary embolism (PE) have been widely reported as postoperative complications of lower limb joint replacement arthroplasty [1, 2]. In particular, PE may be lethal depending on the sites affected and other factors, making its prevention of vital importance. With regard to embolism, risk factors such as advanced age and obesity as well as the components of the thrombus itself like fat, bone marrow, and air have been analysed [3-5], but much remains unclear about the mechanisms promoting venous thromboembolism (VTE) in the postoperative low-

\author{
Corresponding author: \\ Toru Ichiseki \\ Department of \\ Orthopaedic Surgery \\ Kanazawa Medical \\ University \\ Daigaku 1-1 \\ Uchinada-machi \\ Kahoku-gun \\ Ishikawa 920-0293 \\ Japan \\ Phone: +81 76-286-2211 \\ ext. 3214 \\ Fax: +8176 286-4406 \\ E-mail: tsy-ichi@kanazawa- \\ med.ac.jp
}


er limb joint replacement setting, and the primary inciting factor is still obscure. Of these, the underlying pathophysiology of fat embolism syndrome (FES) caused by the release of fat emboli is still unclear. FES is characterised by abrupt worsening of respiratory function with signs such as hypoxaemia, impaired consciousness, haemodynamic insufficiency, and, with fulminant, development of acute respiratory distress syndrome (ARDS), an extremely dangerous disorder in which shock can proceed to death. Although FES most commonly develops after trauma, it has also been reported as a postoperative complication after orthopaedic surgery [6]. When treatment is delayed it can be a highly lethal condition, and thus requires immediate attention $[7,8]$.

In total hip arthroplasty (THA) and total knee arthroplasty (TKA), because the presence of echogenic emboli and snowstorm images identified intraoperatively by transoesophageal echocardiography (TEE) has been associated with the development of PE and DVT [9-13], intraoperative TEE is being increasingly used to clarify the developmental mechanisms of postoperative VTE. In contrast, because the incidence of PE and DVT in upper limb surgery is low and these cases are considered to be low risk, early ambulation and active movement may suffice as prophylactic interventions. However, with total shoulder arthroplasty (TSA) and reverse shoulder arthroplasty (RSA), humeral head replacement (HHR), new-onset DVT, and PE have been reported as postoperative complications [14-16]. Becuase VTE has also been reported in upper limb surgery, despite being generally considered to be of low risk, we undertook the present investigation using TEE to determine the presence of intraoperative echogenic emboli and snowstorm images known to be risk factors. We compared the intraoperative findings in the right atrium between RSA and THA. Moreover, to screen for DVT we measured D-dimer values and compared any changes in this parameter in the perioperative period between RSA and THA.

\section{Material and methods}

The subjects comprised five shoulders undergoing RSA (RSA group) at our institution. There were two men and three women, none of whom had experienced any cardiovascular events preoperatively. Their mean age was 78.4 years in the RSA group. TEE was performed in the supine position. Monitoring was continued from the start of surgery until its completion. The TEE monitoring was done using ME Bicaval, with the right atrium evaluated. In all cases elastic stockings were worn intraoperatively and postoperatively, a foot pump was attached, and edoxaban (DAIICHI SANKYO COMPANY, Tokyo) was administered for 14 days after removal of the drains. In addition, within
2 weeks postoperatively, lower limb venous ultrasonography and lung ventilation-perfusion scintigraphy were performed in all cases. As investigated items, the findings on intraoperative TEE images, mean surgery time, mean intraoperative blood loss, and blood D-dimer values measured intraoperatively, and at postoperative day 1, day 4, 1 week, and 2 weeks were determined. The five hips subjected to THA (THA group) were investigated with the same items and received the same after-treatment, and then compared with RSA group. RSA and THA groups were treated by the same orthopaedic surgeons, and all cases in both groups had TEE performed intraoperatively. In the THA group TEE was performed in the lateral position. All subjects gave their informed consent for inclusion before they participated in the study.

\section{Statistical analysis}

The D-dimer values are presented as mean \pm SE. Significant differences in the D-dimer values intraoperatively, postoperatively, mean operation time, and mean intraoperative blood loss between groups, were assessed using one-way analysis of variance followed by Fisher's protected least significant difference post hoc test. Significance was defined as $p<0.05$.

\section{Results}

Echogenic emboli and snowstorm images were noted on TEE to a similar extent at the time of bone reaming and artificial joint insertion in the right atrium during the intraoperative period in both RSA and THA groups (Figure 1). Especially noteworthy was the finding in both groups of markedly echogenic emboli at the time of stem insertion. The mean surgery time was 78.8 min in RSA group and $64.2 \mathrm{~min}$ in the THA group. The mean intraoperative blood loss was $148 \mathrm{ml}$ in the RSA group and $182 \mathrm{ml}$ in the THA group. D-dimer values in the RSA group were: intraoperative $1.0 \pm 0.2 \mu \mathrm{g} /$ $\mathrm{ml}$, postoperative day $16.0 \pm 0.8 \mu \mathrm{g} / \mathrm{ml}$, postoperative day $43.5 \pm 0.5 \mu \mathrm{g} / \mathrm{ml}$, postoperative week 1 $5.4 \pm 0.5 \mu \mathrm{g} / \mathrm{ml}$, and postoperative week $25.9 \pm 0.3$ $\mu \mathrm{g} / \mathrm{ml}$. In the THA group they were intraoperative $0.6 \pm 0.3 \mu \mathrm{g} / \mathrm{ml}$, postoperative day $14.0 \pm 1.3 \mu \mathrm{g} /$ $\mathrm{ml}$, postoperative day $43.4 \pm 0.9 \mu \mathrm{g} / \mathrm{ml}$, postoperative week $16.6 \pm 0.7 \mu \mathrm{g} / \mathrm{ml}$, and postoperative week $25.3 \pm 1.4 \mu \mathrm{g} / \mathrm{ml}$, with no significant differences found between the two groups (Table I). Also, in neither group was DVT or PE detected by either lower limb venous ultrasonography or lung ventilation-perfusion scintigraphy.

\section{Discussion}

It has long been suggested that the echogenic emboli seen on TEE are related to postoperative 

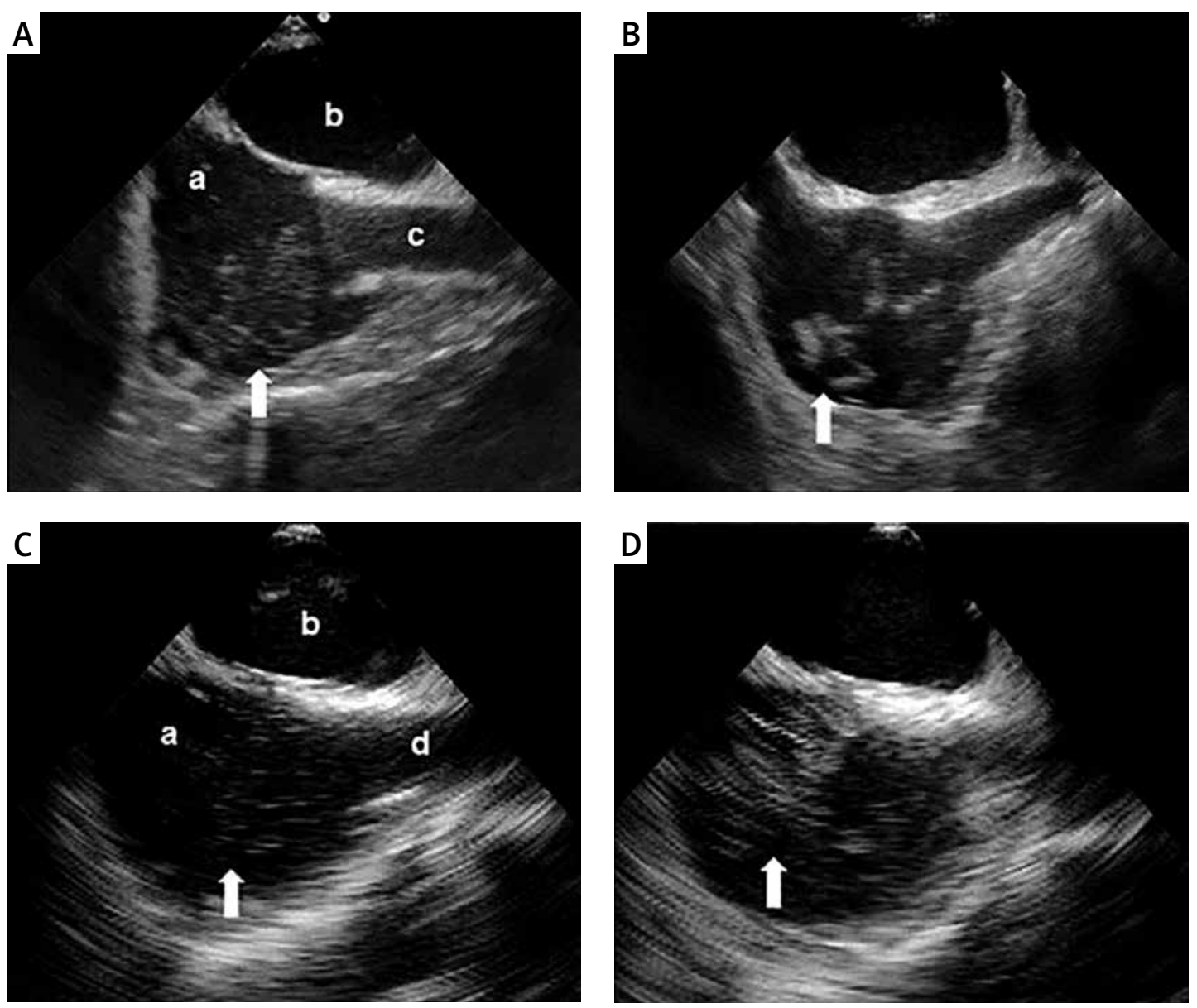

Figure 1. TEE images in RSA and THA. A - In RSA a snowstorm image (white arrow) was observed in the right atrium. B - In RSA echogenic emboli (white arrow) were found in the right atrium. C - In THA a snowstorm image (white arrow) was similarly observed in the right atrium. D - In THA echogenic emboli (white arrow) were similarly found in the right atrium

$a$-right atrium, $b$-left atrium, $c$-superior vena cava, $d$-inferior vena cava

PE and DVT $[5,6,9]$. These emboli arise from the fat and bone marrow released at the time of bone reaming and artificial joint insertion, which cause endothelial damage around the venous system, resulting in the formation of secondary emboli that become the cause of PE $[7,13]$. In contrast, Moriyama et al. reported that intraoperative TEE cannot predict the generation of PE on the first postoperative day [13]. In the present study the echogenic emboli detected on intraoperative TEE were seen in both RSA and THA groups at the time of reaming and artificial joint insertion. Lu et al. demonstrated that in TKA the echogenic emboli observed on intraoperative TEE are taken up transvenously and consist of lipid droplets and bone marrow tissue [12]. Namely, they are thought to consist of lipid droplets and bone marrow tissue generated at the time of bone reaming and

Table I. Changes in intraoperative and postoperative D-dimer values, mean operation time, and mean intraoperative blood loss in each group

\begin{tabular}{|llll|}
\hline Parameter & RSA & THA & $P$-value \\
\hline Mean operation time $[\mathrm{min}]$ & 78.8 & 64.2 & 0.288 \\
\hline Mean intraoperative blood loss $[\mathrm{ml}]$ & 148 & 182 & 0.636 \\
\hline Intraoperative D-dimer $[\mu \mathrm{g} / \mathrm{ml}]$ & $1.0 \pm 0.2$ & $0.6 \pm 0.3$ & 0.223 \\
\hline Postoperative day 1 D-dimer $[\mu \mathrm{g} / \mathrm{ml}]$ & $6.0 \pm 0.8$ & $4.0 \pm 1.3$ & 0.226 \\
\hline Postoperative day 4 D-dimer $[\mu \mathrm{g} / \mathrm{ml}]$ & $3.5 \pm 0.5$ & $3.4 \pm 0.9$ & 0.915 \\
\hline Postoperative week 1 D-dimer $[\mu \mathrm{g} / \mathrm{ml}]$ & $5.4 \pm 0.5$ & $6.6 \pm 0.7$ & 0.167 \\
\hline Postoperative week 2 D-dimer $[\mu \mathrm{g} / \mathrm{ml}]$ & $5.9 \pm 0.3$ & $5.3 \pm 1.4$ & 0.608 \\
\hline
\end{tabular}


artificial joint insertion. FES is characterised by tissue capillary vessel injury caused by the release of lipid droplets from the bone marrow, which in turn induce inflammation and a thrombotic tendency. Intramedullary fat is broken down by tissue lipase into high concentrations of glycerol and toxic free fatty acids that directly cause tissue injury. In the lungs, lung cells and lung epithelial cells sustain damage, which in turn induces vasogenic or cytotoxic edema and haemorrhage. Injured lung epithelium activates a cascade of inflammatory cytokines that may culminate in ARDS [8]. Because of differences in the underlying pathogenetic mechanisms, it can be understood that FES resulting from fat emboli is not the same as PE, in which emboli directly clog the pulmonary vasculature secondary to DVT. PE can be caused by various embolic particles. Postoperative PE occurs secondarily to DVT in the majority of cases, whereas the causes of PE due to fat emboli have yet to be reported clearly. Accordingly, we consider that the occurrence of postoperative PE cannot be predicted from the TEE findings. Namely, there is a strong possibility that among PE reported as a complication of joint arthroplasty FES is also included. In this study, the intraoperative TEE imaging findings documented right atrium echogenic emboli and snowstorm images in the RSA group to a similar in degree to those in the THA group. Based on the above, it is important that when findings of hypoxaemia and PE of unknown origin are noted after joint arthroplasty the possible involvement of FES is considered. Namely, in upper limb surgery the possible occurrence of postoperative FES must be kept in mind. Because no prophylactic strategies have yet been established for FES, when signs of hypoxaemia are evident not only in upper limb surgery but also after joint arthroplasty, adequate postoperative management that takes into account the possibility of FES should be implemented. In upper limb surgery the incidence of PE and DVT is considered low, but the possible postoperative complication of FES should be remembered.

The occurrence of DVT in upper limb surgery has also been described $[15,16]$. In the present study, a prophylactic regimen similar to that used for lower limb joint arthroplasty was adopted postoperatively, with DVT not occurring in either group. However, postoperative D-dimer values did not differ significantly between the groups. With respect to postoperative $D$-dimer values, since RSA showed similarly elevated values to those of THA, like in THA, the possibility of postoperative thrombus development cannot be excluded. On the other hand, since D-dimer is a cross-linked fibrin appearing when fibrin is broken down by plasmin, it is increased in secondary hyperfibrinolysis. For this reason, it may also be increased in conditions inducing signs of secondary hyperfibrinolysis (malignant tumours, inflammatory disorders, infection), in which it is susceptible to the influence of postoperative haematoma and other factors. In this way, elevated values may also be seen in conditions other than DVT, representing false negatives. Accordingly, even though measurement of D-dimer values is useful for screening purposes, any changes noted in them are of greater significance than the values themselves. When postoperative D-dimer values show a rapid increase, the possibility of DVT must be considered. In this study, postoperative D-dimer values showed similar changes in RSA and THA. Since a certain risk of DVT and PE in THA is well recognised and DVT has also been documented in occasional cases undergoing upper limb surgery, we concluded that postoperative changes in D-dimer values should be assessed in RSA like in THA to screen for new-onset DVT.

In conclusion, in the RSA group, echogenic emboli and snowstorm images were detected by TEE to the same extent as in THA. In upper limb surgery the possibility of FES as a postoperative complication should also be remembered. Moreover, because D-dimer values in RSA showed similar changes to those in THA, in upper limb surgery measurements of D-dimer are thought to be useful also in the early detection of postoperative DVT. We suggest that further larger studies be conducted to confirm and support the findings.

\section{Acknowledgments}

Shusuke Ueda and Toru Ichiseki contributed equally to this work.

This study received a grant-in-Aid for Scientific Research from the Ministry of Education, Culture, Sports, Science, and Technology of Japan (no. 24592287), the Ministry of Health, Labour, and Welfare of Japan.

\section{Conflict of interest}

The authors declare no conflict of interest.

\section{References}

1. Fujita S, Hirota S, Oda T, Kato Y, Tsukamoto Y, Fuji T. Deep venous thrombosis after total hip or total knee arthroplasty in patients in Japan. Clin Orthop Relat Res 2000; 375: 168-74.

2. Nagase $Y$, Yasunaga $H$, Horiguchi $H$, et al. Risk factors for pulmonary embolism and the effects of fondaparinux after total hip and knee arthroplasty: a retrospective observational study with use of a national database in Japan. J Bone Joint Surg Am 2011; 93: e146.

3. Tashjian RZ, Lilly DT, Isaacson AM, et al. Incidence of and risk factors for symptomatic venous thromboembolism after shoulder arthroplasty. Am J Orthop (Belle Mead NJ) 2016; 45: E379-85. 
4. Parvizi J, Huang R, Raphael IJ, Arnold WV, Rothman RH. Symptomatic pulmonary embolus after joint arthroplasty: stratification of risk factors. Clin Orthop Relat Res 2014; 472: 903-12.

5. Memtsoudis SG, Besculides MC, Gaber L, et al. Risk factors for pulmonary embolism after hip and knee arthroplasty: a population-based study. Int Orthop 2009; 33: 1739-45.

6. Walker NM, Bateson T, Reavley P, Prakash D. Fatal fat embolism following femoral head resection in total hip arthroplasty. Hip Int 2008; 18: 332-4.

7. Taviloglu K, Yanar H. Fat embolism syndrome. Surg Today 2007; 37: 5-8.

8. Kosova E, Bergmark B, Piazza G. Fat embolism syndrome Circulation 2015; 131: 317-20.

9. Pitto RP, Hamer H, Fabiani R, Radespiel-Troeger $M$, Koessler $M$. Prophylaxis against fat and bone-marrow embolism during total hip arthroplasty reduces the incidence of postoperative deep-vein thrombosis: a controlled, randomized clinical trial. J Bone Joint Surg Am 2002; 84-A: 39-48.

10. Walker P, Bali K, Van der Wall H, et al. Evaluation of echogenic emboli during total knee arthroplasty using transthoracic echocardiography. Knee Surg Sports Traumatol Arthrosc 2012; 20: 2480-6.

11. Zhao J, Zhang J, Ji X, Li X, Qian Q, Xu Q. Does intramedullary canal irrigation reduce fat emboli? A randomized clinical trial with transesophageal echocardiography. J Arthroplasty 2015; 30: 451-5.

12. Lu K, Xu M, Li W, Wang K, Wang D. A study on dynamic monitoring, components, and risk factors of embolism during total knee arthroplasty. Medicine (Baltimore) 2017; 96: e9303.

13. Moriyama M, Watanabe S, Hiraki T, Kano T, Okawa T, Ishibashi M. Relationship between intraoperative transoesophageal echocardiography findings and perfusion lung scintigraphy results on first postoperative day. $\mathrm{Br}$ J Anaesth 2005; 94: 607-12.

14. Sperling JW, Cofield RH. Pulmonary embolism following shoulder arthroplasty. J Bone Joint Surg Am. 2002; 84-A: 1939-41.

15. Jiang JJ, Toor AS, Shi LL, Koh JL. Analysis of perioperative complications in patients after total shoulder arthroplasty and reverse total shoulder arthroplasty. J Shoulder Elbow Surg 2014; 23: 1852-9.

16. Young BL, Menendez ME, Baker DK, Ponce BA. Factors associated with in-hospital pulmonary embolism after shoulder arthroplasty. J Shoulder Elbow Surg 2015; 24: e271-8. 